\title{
An Optimizing On-duty Scheduling of Air Traffic Controller Consid- ering Fatigue Factors
}

\author{
Yonggang Yan, Guozhuang Pan and Zhiyuan Shen ${ }^{+}$ \\ College of Civil Aviation, Nanjing University of Aeronautics and Astronautics, Nanjing, China
}

\begin{abstract}
As the rapid development of the world's civil aviation and the rapid increase of flights in recent years, the fatigue of the controllers has become a challenges in the field of civil aviation safety management. The state-of-the-art on-duty scheduling methods are hardly considering the ATC fatigue. Firstly, this paper introduces the definition of fatigue and its influence factors. Then, the causes of fatigue and the influence of on-duty scheduling based on ATC fatigue are emphatically analysed. Then, the mode and restrictive factors of on-duty scheduling are explained. Finally, according to the collected data from area zone control unit in Xinjiang Bureau of CAAC, an optimize Scheduling for Air Traffic Controller is given. This paper provides the control unit theoretical guidance to the analysis and mitigation of the controllers fatigue, and further enriches the theory of the fatigue management of the controllers.
\end{abstract}

Keywords: Air Traffic Controller, Fatigue, Scheduling Optimization.

\section{Introduction}

With the rapid development of China's civil aviation, the air traffic flow continues to increase, and the civil aviation industry has suffered from more problems. Air traffic controllers play an extremely important role in keeping flight safety. With the increase of the number of flights, controllers are facing increasing complex airspace environment and workload.

The essential purpose of the related research on fatigue is to explore how to alleviate fatigue. Gruz et al. assessed napping patterns under 45 minutes and 2 hours, pointing out that both napping patterns can improve cognitive efficiency, and 2 hours sleep has better effect; napping in midnight shift is the most helpful to improve efficiency, and sleepiness will be reduced accordingly [1]. Another empirical study assessed the impact of 40-minute naps on energy efficiency and alertness of controllers on early midnight duty (22:30-06:00) and late midnight duty (23:30-06:30). The results show that during the last hours of the controller shift, napping reduces spectral energy and the likelihood of slow eye movement [2]. In addition, it can reduce the impact of fatigue that a weekly cycle, a fast counterclockwise cycle, and a long, continuous midnight nap on duty. Some studies have found that increasing stimulating factors in work can alleviate mental fatigue and enhance alertness. By researching, the techniques of vigilance enhancement include physical regulation and chemical regulation [3].Physical regulation method mainly includes somatosensory stimulation such as sound, light, electricity, magnetism, temperature and vibration. External stimulation can alleviate the degree of fatigue and improve the alertness of the brain in a short time [4].

This research starts with the analysis of the fatigue factors of the controller, and then according to the actual work situation of a control unit, some suggestions are given to optimize the scheduling of controllers. This paper provides the control unit theoretical guidance to the monitoring and mitigation of the controllers fatigue, and further enriches the theory of the fatigue management of the controllers.

\section{Controller Fatigue and Scheduling}

Corresponding author. Tel.: +86 13951916587

E-mail address: shenzy@nuaa.edu.cn 
The definition about fatigue given by Dinges in 1995 is that fatigue refers to a neurobiological process directly related to the individual's physiological sleep needs and the physiological pacemaker in the brain [5]. In the field of transportation, fatigue is also one of the most concerned areas of scholars. International Maritime Organization defines fatigue as: due to excessive psychological or physical consumption, reducing physical and psychological ability, impairing almost all performance, including limb movement speed, limb strength, decision-making ability, coordination ability and reflection time [6]. The International Civil Aviation Organization (ICAO) defines fatigue as a physiological state in which the ability to perform mental or physical activities decreases due to insufficient sleep, prolonged wakefulness, circadian rhythm or excessive workload (mental and/or physical activity), which impairs the alertness of crew members and their ability to safely operate aircraft or perform safety-related duties. Ability [7].

For the controllers, they shoulder the great responsibility of airplane and life safety. They are facing the pressure of work under high flow. On the one hand, high-intensity work brings physical and mental pressure; on the other hand, the controllers' shift work will directly cause sleep loss and destroy the circadian rhythm of the human body. This irregular work further increases the pressure, which leads to fatigue easily. The efficiency and vigilance of night-shift controllers are at the lowest level throughout the day, so shifting is a very important factor leading to fatigue.

Scheduling is defined as the standard time for each employee to go to and from work every day in a period, and decides whether to rest every day, whether public holidays are effective or not, whether public holidays are postponed or not. The so-called scheduling problem is the process of generating a working schedule that meets certain constraints over a period of time according to the work plan [8]. Scheduling should conform to the rules and regulations, pay attention to fairness, achieve more rest time after duty (as far as possible, the time for duty and rest is block), less tasks, less transactional, high hourly fees, and can take into account other matters. Fair and reasonable scheduling results are of great significance for mobilizing staff's enthusiasm and improving work efficiency, and can bring enormous economic benefits.

It is needed that considering important shifts and those with strong control ability. Then adjust the balance with other shifts requiring lower requirements and more staff. When dealing with the balance of controllers, the first step is to distribute the people with relatively weak ability and low current control level, and then to adjust the balance with the people with strong ability. When dealing with the balance of night shift staff, we should first allocate the low ability, and then adjust the balance with the strong ability.

\section{Optimized Scheduling}

\subsection{Current scheduling}

Following is an analysis of the actual scheduling of an area control room in Xinjiang. The characteristics of the air traffic control work of the control room are that the main flights are in and out of the port during the day and international overflights at night. The 24-hour uninterrupted air traffic control service is implemented. Combining airspace conditions, flight flow, number of controllers and long-term habits, the whole day is divided into four classes, six hours each, namely: 0200-0800 for night, 0800-1400 for morning, 1400-2000 for medium, 2000-next day 0200 for night. The control room is divided into four crews for four shifts, each crew four days a round, the first day of mid-shift, the second day of early overnight shift. The third night voyage, the fourth day of rest, that is, on three breaks. Because the night flight volume is relatively small, the number of people is relatively small, but because of the biological clock, night crew must be guaranteed first, and then night voyage crew should be considered. The crew will arrange night crew and night voyage crew separately. Normally, night crew will not be on night voyage crew and night voyage crew will not be on night crew. Affected by flight flow and personnel conditions, there will also be continuous night voyage crew after night crew. As shown in Table 1, controllers in the team can be matched with green filling color, but not red filling color. A-L means different controllers respectively where A and B are Grade 09 teacher, $\mathrm{C}$ and D are Grade 11 teacher; $\mathrm{E}$ and $\mathrm{F}$ are Grade 2 controller; $\mathrm{G}$ is Grade 3 controller; H, I and J are Grade 5 controller; $\mathrm{K}$ and $\mathrm{L}$ are probationary controller. 
Tab.1: Team Personnel Matching.

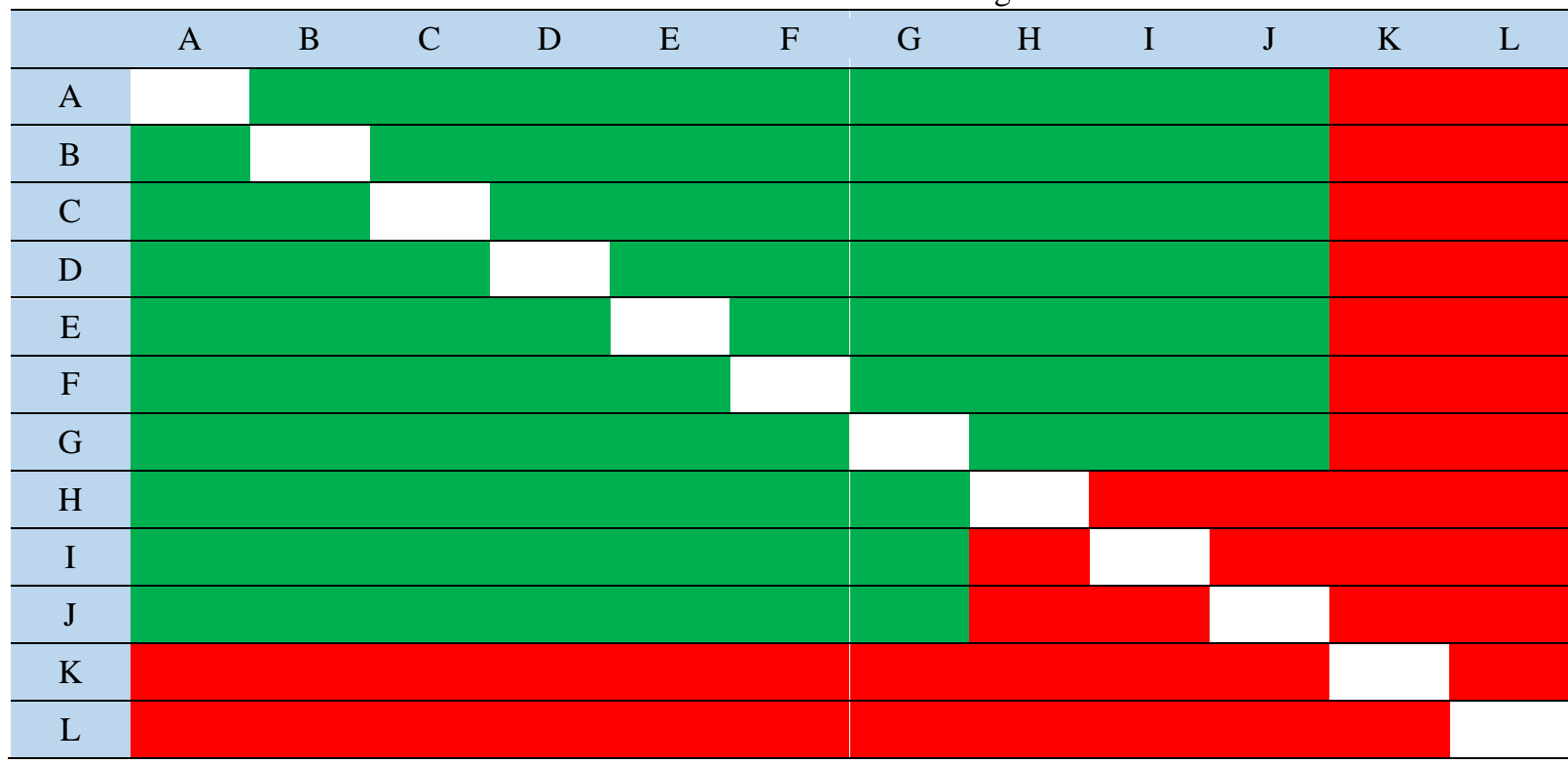

(1) Fore-shift arrangement

Nine billing controllers will be on duty in the morning. In the morning 08:00, four controllers will receive sector AR02/AR03 and AR05/AR06. One dynamic billing controllers (level 16 controllers) will take dynamic seats, and 6 controllers (having breakfast) will replace sector AR03 and AR05/AR06 at 08:30, respectively. They will also open sector AR02 seats (AR02/AR03 sub-fans) and be replaced by controllers to eat. At 09:00 controllers take over the dynamic seats to eat. One controller joins sector AR02 and AR03 rotation. The other two controllers divide sector AR05/AR06 into AR05 and AR06, and then there will be a rotating.

(2) Intermediate shift arrangement

Nine L/C controllers and one dynamic controller are on duty at mid-shift. Sector AR02/AR03 arrange five people, each half-hour rest rotation replacement, while sector AR05/AR06 arrange four people. The principle of each whole point as a node to split and merge the sector, after each two people rest half an hour to re-divide the sector, specific split and close the sector depending on the flight flow at that time fine-tuning. ADS-C seats are open according to circumstances and do not require 24-hour duty.

(3) Night voyage arrangement

Night Airlines arranges five billing controllers on duty, seats AR02/AR03 merge sector, while Sector AR05/AR06 merge operation. Each person's half-hour elaboration time is replaced in turn. A controller is on duty for dynamic seats and temporary ADS-C seats.

(4) Night shift arrangement

Four controllers will be on duty at night, separate or merge sectors at the right time. One controller will be on duty for dynamic seats and one controller will be on temporary duty for ADS-C seats.

The actual scheduling of the control room is shown in Tab.2. The red in the column of "leading class" is to control the management performance personnel, that is, the Department leaders, according to the situation "fill vacancies", such as "A"; the red in the column of "Control" is to "connect" night flights, such as "G" 19 morning, night flights, 20 night flights.

Tab.2: Control Room Duty Schedule.

\begin{tabular}{|c|c|c|c|c|c|c|}
\hline Date & Post & Fore & Middle & $\begin{array}{c}\text { Night Voy- } \\
\text { age }\end{array}$ & Night & Spare Time \\
\hline \multirow{6}{*}{1} & Leading & $\mathrm{A}$ & $\mathrm{B}$ & $\mathrm{A}$ & $\mathrm{C}$ & \\
\hline & Dynamic & $\mathrm{D}$ & $E$ & $\mathrm{~F}$ & $\mathrm{D}$ & \\
\hline & \multirow{4}{*}{ Control } & $G$ & $\mathrm{O}$ & $X$ & $G$ & \multirow{4}{*}{ e } \\
\hline & & $\mathrm{H}$ & $\mathrm{P}$ & $\mathrm{Y}$ & I & \\
\hline & & I & Q & $\mathrm{Z}$ & $\mathrm{d}$ & \\
\hline & & $\mathrm{J}$ & $\mathrm{R}$ & $\mathrm{a}$ & $\mathrm{N}$ & \\
\hline
\end{tabular}




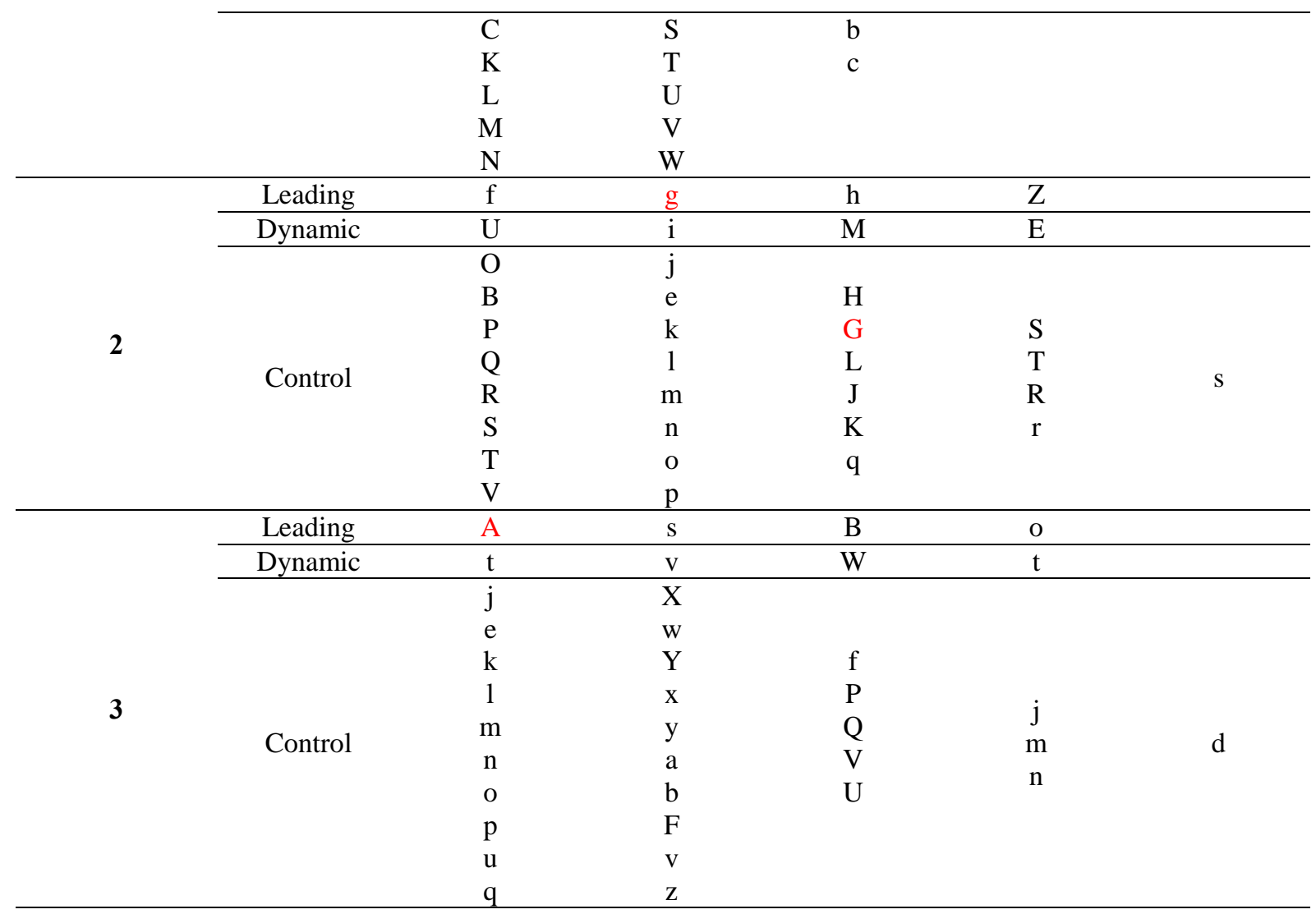

Where $\mathrm{A}-\mathrm{Z}$ and a-Z are 48 different controllers.

\subsection{Suggestions on Scheduling Optimization}

Under the circumstances that the fatigue and fairness of controllers are paid more and more attention, the purpose of motivating employees' work enthusiasm can be achieved by complying with the requirements of civil aviation management regulations, conforming to various regulations and rules, high income of controllers, striving for equal workload of each controllers, and effective and accurate scheduling. The purpose of labor.

(1) At present, scheduling is manual, with many constraints and changes in shifts and personnel, which will not be considered thoroughly and imperfectly. For example, in a certain case, because the AA has to deploy personnel training, plus leave to go out and so on, it took 6 hours for manual scheduling to barely meet the requirements of guaranteeing post duty, and other requirements such as fairness have not yet been taken into account.

(2) Adequate restorative sleep after night shift. After the night shift, we must arrange enough time to rest. After the night shift 0800 , we will overlap posts, give comments after the shift, wash properly, have breakfast, and begin to rest after 0830. At least eight consecutive hours of rest, after 1600 p.m. With lunch and some noise during the day, rest will be greatly affected. So we must not attend meetings, training and other activities after night shift.

(3) Night naps. Physiology divides people's sleep into five stages. It is easy to wake up, maintain the efficiency and alertness of the body, improve the productivity, creativity and memory of people, and have the effect of eliminating fatigue during the first stage about 20 minutes. At present, the control unit strictly enforces the principle that "continuous duty time should not exceed 2 hours, and the interval between two duty times should not be less than 30 minutes". The 30-minute rest time in the middle can just go to the bathroom and complete a 20-minute nap. This time try not to play with mobile phones, eat dinner, to avoid sleepiness after rest.

(4) Regular work and rest habits. Chaotic physiological rhythm is an important cause of controller fatigue. Regular life is not only not easy to fatigue, but also helpful to develop good working and living habits. The biological clock can also adjust itself according to the rules. From the scheduling, temporary arrangements should be minimized and activities that need to be attended should be notified as early as possible. 
Based on the analysis of the fatigue factors of the controllers, the fatigue knowledge is deeply understood, the team scheduling of the control room is analyzed and optimized, and the good working and sleeping mode of the controllers is recommended. Only by establishing reasonable, scientific and humanized management of the controllers' shift can the fatigue management of the controllers be effectively implemented and the safety of air traffic control be further guaranteed.

\section{Conclusion}

In recent years, China's civil aviation industry has developed substantially and the number of flights has increased dramatically, which puts forward higher requirements for controllers. Controller's work has a great impact on all aspects of the civil aviation industry, such as aviation safety, flight delays and so on. The fatigue problem of controllers will directly affect the performance of controllers in their work, and will seriously threaten the safety of the civil aviation industry. Firstly, this paper introduces the definition of fatigue and the influence of controller fatigue. Then, the causes of controller fatigue are analyzed, and the influence of shift scheduling on controller fatigue is emphatically analyzed. Then, the mode and restrictive factors of shift scheduling are explained. Finally, according to the scheduling situation of an area control room in Xinjiang, some suggestions are given to optimize the scheduling. This paper provides the control unit theoretical guidance to the analysis and mitigation of the controllers fatigue, and further enriches the theory of the fatigue management of the controllers.

\section{Acknowledgements}

The authors would like to thank the financial support by the Open Fund of Graduate Innovation Base (Laboratory) of Nanjing University of Aeronautics and Astronautics funded project (kfjj20180724).

\section{Reference}

[1] Cruz C, Della R P, Hackworth C. Effects of quick rotating shift schedules on the health and adjustment of air traffic controllers [J]. Aviation Space \& Environmental Medicine, 2000, 71(4):400.

[2] Signal T L, Gander P H, Anderson H, et al. Scheduled napping as a countermeasure to sleepiness in air traffic controllers [J]. Journal of Sleep Research, 2009, 18(1):11-19.

[3] Zhu Xianfeng, Liu Yating, Zhang Yi, et al. Research status and progress of methods to improve brain alertness [J].Journal of Biomedical Engineering, 2013 (3): 651-655.

[4] Schmidt E A, Schrauf M, Simon M, et al. The short-term effect of verbally assessing drivers' state on vigilance indices during monotonous daytime driving [J]. Transportation Research Part F Traffic Psychology \& Behaviour, 2011, 14(3):0-260.

[5] Cebola N A, Kilner A B. When are you too tired to be safe? Exploring the construction of a Fatigue Index in atm [J]. Retrieved April, 2010.

[6] MSC/Circ. 1014Guidance on fatigue mitigation and management[C]. London: IMO, 2001.

[7] Mason G. Fatigue risk management systems [J]. Log, 2009.

[8] Li Qing, Zhang Jun, Zhang Xuejun, et al. Multi-objective optimization model and algorithm for scheduling problem [J]. Journal of Beijing University of Aeronautics and Astronautics, 2003, 29 (9). 